\title{
Optimal Placement of Piezoelectric Plates to Control Multimode Vibrations of a Beam
}

\author{
Fabio Botta, ${ }^{1}$ Daniele Dini, ${ }^{2}$ Christoph Schwingshackl, ${ }^{2}$ Luca di Mare, ${ }^{2}$ and Giovanni Cerri ${ }^{1}$ \\ ${ }^{1}$ Dipartimento di Ingegneria, Università degli Studi Roma Tre, Via della Vasca Navale, 79-00146 Roma, Italy \\ ${ }^{2}$ Department of Mechanical Engineering, Imperial College London, Exhibition Road, London SW7 2AZ, UK
}

Correspondence should be addressed to Fabio Botta; fabio.botta@uniroma3.it

Received 14 July 2013; Revised 11 October 2013; Accepted 21 October 2013

Academic Editor: Marc Thomas

Copyright (C) 2013 Fabio Botta et al. This is an open access article distributed under the Creative Commons Attribution License, which permits unrestricted use, distribution, and reproduction in any medium, provided the original work is properly cited.

Damping of vibrations is often required to improve both the performance and the integrity of engineering structures, for example, gas turbine blades. In this paper, we explore the possibility of using piezoelectric plates to control the multimode vibrations of a cantilever beam. To develop an effective control strategy and optimize the placement of the active piezoelectric elements in terms of vibrations amplitude reduction, a procedure has been developed and a new analytical solution has been proposed. The results obtained have been corroborated by comparison with the results from a multiphysics finite elements package (COMSOL), results available in the literature, and experimental investigations carried out by the authors.

\section{Introduction}

The vibration control is a problem of great interest in many engineering fields since it allows avoiding problems connected with the vibrations. Blade vibrations in aircraft engines, for example, are often induced by interactions between blades and fluid and the associated fatigue phenomena can give rise to catastrophic failures [1-3]. Typically, passive damping systems, such as friction damping, are used to increase the blade life. These systems are very effective, but, in contrast to active damping elements, they are not able to change their characteristics depending on the system response. In the last two decades, the adoption of piezoelectric elements has received considerable attention by many researchers for its potential applicability to different areas of mechanical, aerospace, aeronautical, and civil engineering. These elements have an interesting coupling between electrical and mechanical properties: a deformation appears when an electric field is applied and vice versa $[4,5]$. Their effectiveness to damp a particular excited mode or a multimode combination strongly depends on their position; in fact the study of their optimal position has received increasing attention. Typically, the aim of these studies is to find the position that minimizes an objective function or maximizes the degree of modal controllability (see $[6,7]$ for a review). The first study concerned with the optimal position to damp a specified mode has been that of Crawley and de Luis [4]. They found that the actuators should be in regions of higher average strain. Analogous results have been found by other researchers $[8,9]$. For a cantilever beam Sunar and Rao [10], Demetriou [11], and Bruant et al. [12] found that the closer the piezoelectric actuators are to the fixed end, the more efficient they are. For a simply supported beam Yang et al. [13] found that, to control one specific mode, the optimal position for the piezoelectric plates is within the regions separated by the vibration nodal lines. In the paper by Barboni et al. [14] the possibility of exciting the flexural dynamics of an Euler-Bernoulli beam, according to a single mode, is examined. The results show that, to excite a desired mode, the actuator must be placed between two consecutive points at which the curvature becomes zero. Aldraihem et al. [15] studied the optimal length and location for different boundary conditions. They investigated beams with one pair and two pairs of piezoelectric actuators. Their optimization criterion was based on beam modal cost and controllability index; moreover, they added a penalty term to consider the actuator length (and the cost, weight, and space factors associated to this). Baz and Poh [16] studied the effects of varying the thickness and material of the bonding layer as well as the position of the piezoelectric actuators. 
They consider three beam elements to model a cantilever beam and the results show that it is preferable to place the actuator in a region of large strain. Subsequently Yang and Lee presented an analytical model for simultaneous optimization of noncollocated [17] and collocated [18] piezoelectric sensor/actuator placement and feedback control gain. The results show that this procedure can avoid the instability of the structural control system. Q. Wang and C. M. Wang [19] studied modal and multimodal vibrations. They propose a new controllability index and illustrated various beam examples with a pair of collocated piezoelectric actuators. More recently, studies about their use in blades of turbomachinery have been carried out; however, only few of these concern active damping [20-23]. Unfortunately in many real cases the loads applied to the structure excite more than one mode, with different amplitudes, and the excited modes can change during service. Therefore, the implementation of an active system that is capable of changing the work configuration of the piezoelectric plates can increase considerably their efficiency in the damping of the vibrations. In this paper, a new function to find the optimal placement of piezoelectric plates to control the multimode vibrations of the cantilever beam, with different amplitudes of the single modes, is defined. An analytical solution is proposed and the results are also compared with the FEM simulations and results from the literature with very good agreement.

\section{Governing Equations for Piezoelectric Coupled Beam}

In Figure 1 an Euler-Bernoulli cantilever beam with attached piezoelectric patches is schematically shown; the two PZT plates are applied in a symmetrical position with respect to the mid plane. Indicating by $\delta L_{e}, \delta L_{\text {in }}$, and $\delta L_{a}$ the virtual work of the elastic, inertial, and piezoelectric forces, respectively, the principle of the virtual work can be written as (in the following, the PZT plates will be considered perfectly bonded to the structure, their mass and inertia negligible with respect to the mass and inertia of the beam, and their thickness very lower than the thickness of the beam)

$$
\delta L_{e}+\delta L_{\mathrm{in}}+\delta L_{a}=0
$$

Using the pin force model [4] the action of the PZT plates can be modeled by two flexural moments concentrated at the end of the plates (Figure 1) with

$$
\begin{gathered}
M(t)=\frac{\psi}{6+\psi} E_{a} c T_{a} T_{b} \Lambda(t), \\
\Lambda(t)=\frac{d_{31}}{T_{a}} V(t) \\
\psi=\frac{E_{b} T_{b}}{E_{a} T_{a}}
\end{gathered}
$$

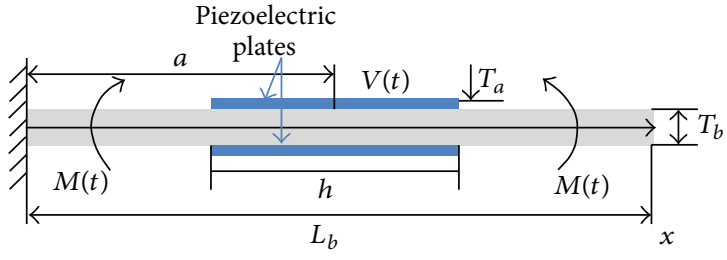

FIGURE 1: Reference configuration and action of the PZT plates.

So by indicating $w$ the vertical displacement, the virtual work of the PZT plates can be written as (the virtual quantities are oversigned by a tilde)

$$
\delta L_{a}=M\left(\left.\frac{\partial \widetilde{w}}{\partial x}\right|_{x=a+(h / 2)}-\left.\frac{\partial \widetilde{w}}{\partial x}\right|_{x=a-(h / 2)}\right) .
$$

The variables $a$ and $h$ can vary within the domain:

$$
\begin{gathered}
0 \leq a-\frac{h}{2} \leq L_{b} \\
0 \leq a+\frac{h}{2} \leq L_{b}, \\
0 \leq a \leq L_{b}, \\
0 \leq h \leq L_{b}
\end{gathered}
$$

and their value depends on the modes that must be damped [14].

The virtual work of the elastic and inertial forces can be written, respectively, as

$$
\begin{gathered}
\delta L_{e}=E_{b} I_{b} \int_{0}^{L_{b}} \frac{\partial^{2} w}{\partial x^{2}} \frac{\partial^{2} \widetilde{w}}{\partial x^{2}} d x \\
\delta L_{\text {in }}=-\rho S \int_{0}^{L_{b}} \frac{\partial^{2} w}{\partial t^{2}} \widetilde{w} d x .
\end{gathered}
$$

Using the modal analysis technique, indicating by $\phi_{i}(x)$ the $i$ th flexural modal displacement of the cantilever beam and by $X_{i}(t)$ its amplitude, the vertical displacement will be approximated by

$$
w(x, t)=\sum_{i=1}^{N} X_{i}(t) \phi_{i}(x)
$$

where $N$ is the number of the considered modes.

Substituting the last in (3), (5), considering $\widetilde{w}=\phi_{j}(x)$, and successively in (1), the following governing equation is obtained:

$$
\mathbf{M} \ddot{\mathbf{X}}(t)+\mathbf{K X}(t)=\mathbf{B}(a, h) V(t),
$$

where $\mathbf{X}$ represents the vector of the amplitudes of the modes, $\mathbf{M}$ and $\mathbf{K}$ are, respectively, the mass and the stiffness 
matrix, and $\mathbf{B}(a, h)$ is the vector control (the prime denotes the first spatial derivative):

$$
\begin{aligned}
& \mathbf{B}(a, h) \\
& =\breve{M}\left[\phi_{1}^{\prime}\left(a+\frac{h}{2}\right)-\phi_{1}^{\prime}\left(a-\frac{h}{2}\right),\right. \\
& \phi_{2}^{\prime}\left(a+\frac{h}{2}\right)-\phi_{2}^{\prime}\left(a-\frac{h}{2}\right), \ldots, \\
& \left.\phi_{N}^{\prime}\left(a+\frac{h}{2}\right)-\phi_{N}^{\prime}\left(a-\frac{h}{2}\right)\right]
\end{aligned}
$$

with $\breve{M}=(\psi /(6+\psi)) E_{a} c T_{a} T_{b}\left(d_{31} / T_{a}\right)$.

If the Rayleigh damping is considered, (7) becomes

$$
\mathbf{M} \ddot{\mathbf{X}}(t)+\mathbf{C} \dot{\mathbf{X}}(t)+\mathbf{K X}(t)=\mathbf{B}(a, h) V(t)
$$

with

$$
\mathbf{C}=\alpha \mathbf{M}+\beta \mathbf{K}
$$

Indicating by $\boldsymbol{\omega}$ the diagonal matrix of the natural frequencies of the beam, using the normal modes $(\mathbf{M}=\mathbf{I}, \mathbf{K}=$ $\omega^{2}$ ), and assuming $\beta=0$, (9) becomes

$$
\ddot{\mathbf{X}}(t)+\alpha \dot{\mathbf{X}}(t)+\omega^{2} \mathbf{X}(t)=\mathbf{B}(a, h) V(t)
$$

If a single mode excitation is considered, Barboni et al. [14] found the optimal placement of PZTs. However, in some practical applications, for example, gas turbine blades, the load spectrum can include various modes, each of which is characterized by different amplitudes, and a new strategy needs to be developed. In this paper, the multimode damping will be obtained by applying a counterphase load, by PZTs plates, to the external excitation. The effectiveness of the piezoelectric elements will be measured by the amplitude of the vertical displacement of the free end, so that the most effective (optimal) position, identified in terms of $a$ and $h$ (Figure 1), will be the location which maximizes this amplitude. Therefore, assigning a general spectrum to the PZT load (which corresponds to the spectrum of $V(t)$; see (2))

$$
V(t)=\sum_{j=1}^{N s} V_{j} \cos \left(\omega_{j} t\right)
$$

where $N s$ is the number of excited modes. The solution of (11) becomes
$X_{i}(t)$

$$
\begin{array}{r}
=\sum_{j=1}^{N s}\left[-\frac{e^{-t \alpha / 2} B_{i} V_{j} \cosh \left((1 / 2) t \sqrt{\alpha^{2}-4 \omega_{i}^{2}}\right) \sqrt{\alpha^{2}-4 \omega_{i}^{2}}\left(\omega_{i}^{2}-\omega_{j}^{2}\right)}{\sqrt{\alpha^{2}-4 \omega_{i}^{2}}\left(\alpha^{2} \omega_{j}^{2}+\left(\omega_{i}^{2}-\omega_{j}^{2}\right)^{2}\right)}\right. \\
-\frac{e^{-t \alpha / 2} B_{i} V_{j}\left(\alpha \sinh \left((1 / 2) t \sqrt{\alpha^{2}-4 \omega_{i}^{2}}\right)\left(\omega_{i}^{2}+\omega_{j}^{2}\right)\right)}{\sqrt{\alpha^{2}-4 \omega_{i}^{2}}\left(\alpha^{2} \omega_{j}^{2}+\left(\omega_{i}^{2}-\omega_{j}^{2}\right)^{2}\right)} \\
\left.+\frac{B_{i} V_{j} \alpha \sin \left(\omega_{j} t\right) \omega_{j}}{\alpha^{2} \omega_{j}^{2}+\left(\omega_{i}^{2}-\omega_{j}^{2}\right)^{2}}+\frac{B_{i} V_{j} \cos \left(t \omega_{j}\right)\left(\omega_{i}^{2}-\omega_{j}^{2}\right)}{\alpha^{2} \omega_{j}^{2}+\left(\omega_{i}^{2}-\omega_{j}^{2}\right)^{2}}\right]
\end{array}
$$

It is possible to obtain an approximate form of (13) considering that the $i$ th term is the dominant one (the $i$ th term of $V(t)$ is exciting $X_{i}(t)$ near its resonance). After some algebraic manipulation, (13) can be simplified to

$$
X_{i}(t) \cong \frac{B_{i} V_{i} \sin \left(\omega_{i} t\right)}{\alpha \omega_{i}}
$$

$$
-\frac{e^{-(1 / 2) t\left(\alpha+\sqrt{\alpha^{2}-4 \omega_{i}^{2}}\right)}\left(-1+e^{t \sqrt{\alpha^{2}-4 \omega_{i}^{2}}}\right) B_{i} V_{i}}{\alpha \sqrt{\alpha^{2}-4 \omega_{i}^{2}}} .
$$

The second term of the right hand side of (14) represents the transient part so that, if this is neglected, the amplitude of the displacement of the free end can be written as

$$
|\widehat{w}(a, h)|=\sum_{i=1}^{N}\left|\frac{B_{i}(a, h) V_{i} \phi_{i}\left(L_{b}\right)}{\alpha \omega_{i}}\right| .
$$

In the following a bimodal excitation is considered, indicating by $i_{1}, i_{2}$ the indexes of the first and second considered modes respectively, and $r \leq 1$ the relative contribution to the excitation induced by the second mode with respect to the first one (i.e., $r=1$ corresponds to the second mode governing the excitation without any contribution from the first mode). From (12):

$$
V(t)=(1-r) \cos \left(\omega_{i_{1}} t\right)+r \cos \left(\omega_{i_{2}} t\right) .
$$

Substituting (16) into (15) and introducing

$$
\begin{gathered}
g_{i_{1}}(r, \xi)=\frac{\breve{M}}{\alpha} \frac{1-r}{\omega_{i_{1}}} \phi_{i_{1}}\left(L_{b}\right) \phi_{i_{1}}^{\prime}(\xi), \\
g_{i_{2}}(r, \xi)=\frac{\breve{M}}{\alpha} \frac{r}{\omega_{i_{2}}} \phi_{i_{2}}\left(L_{b}\right) \phi_{i_{2}}^{\prime}(\xi),
\end{gathered}
$$

it is obtained that

$$
\begin{aligned}
|\widehat{w}(r, a, h)|= & \left|g_{i_{1}}\left(r, a+\frac{h}{2}\right)-g_{i_{1}}\left(r, a-\frac{h}{2}\right)\right| \\
& +\left|g_{i_{2}}\left(r, a+\frac{h}{2}\right)-g_{i_{2}}\left(r, a-\frac{h}{2}\right)\right| .
\end{aligned}
$$


Indicating by $\left(\xi_{1}=a+(h / 2), \xi_{2}=a-(h / 2)\right)$, respectively, the position of the right and the left ends of the piezoelectric plates it will be

$$
\begin{aligned}
\left|\widehat{w}\left(r, \xi_{1}, \xi_{2}\right)\right|= & \left|g_{i_{1}}\left(r, \xi_{1}\right)-g_{i_{1}}\left(r, \xi_{2}\right)\right| \\
& +\left|g_{i_{2}}\left(r, \xi_{1}\right)-g_{i_{2}}\left(r, \xi_{2}\right)\right| .
\end{aligned}
$$

If $\left(\widehat{\xi}_{1}, \widehat{\xi}_{2}\right)$ are the coordinates of the absolute maximum of $\left|\widehat{w}\left(r, \xi_{1}, \xi_{2}\right)\right|$, it is possible to write

$$
\begin{aligned}
\left|\widehat{w}\left(r, \xi_{1}, \xi_{2}\right)\right|_{\max }= & \left|\widehat{w}\left(r, \hat{\xi}_{1}, \hat{\xi}_{2}\right)\right|=\left|g_{i_{1}}\left(r, \widehat{\xi}_{1}\right)-g_{i_{1}}\left(r, \widehat{\xi}_{2}\right)\right| \\
& +\left|g_{i_{2}}\left(r, \widehat{\xi}_{1}\right)-g_{i_{2}}\left(r, \widehat{\xi}_{2}\right)\right| .
\end{aligned}
$$

Considering that $g_{k}(r, \xi)$ has, for all the considered values of $k$, its positive absolute maximum at $\widehat{\xi}_{1}=L_{b}$, this value will also be the abscissa of the positive absolute maximum for $\left|g_{i_{1}}\left(r, \xi_{1}\right)-g_{i_{1}}\left(r, \xi_{2}\right)\right|+\left|g_{i_{2}}\left(r, \xi_{1}\right)-g_{i_{2}}\left(r, \xi_{2}\right)\right|$. This implies that the PZT plates should always be placed with their right edges terminating at the tip of the beam independently of the excited modes and value of $r$, and hence

$$
\begin{aligned}
\left|\widehat{w}\left(r, \xi_{1}, \xi_{2}\right)\right|_{\max }= & \left|\widehat{w}\left(r, L_{b}, \widehat{\xi}_{2}\right)\right|_{\max } \\
= & \left|g_{i_{1}}\left(r, L_{b}\right)-g_{i_{1}}\left(r, \widehat{\xi}_{2}\right)\right| \\
& +\left|g_{i_{2}}\left(r, L_{b}\right)-g_{i_{2}}\left(r, \widehat{\xi}_{2}\right)\right| .
\end{aligned}
$$

Moreover, since

$$
0<g_{j}\left(r, L_{b}\right)>g_{j}(r, \xi) \quad \forall \xi \in\left[0, L_{b}\right), \forall j .
$$

(21) becomes

$$
\begin{aligned}
\left|\widehat{w}\left(r, \xi_{1}, \xi_{2}\right)\right|_{\max }= & \left|\widehat{w}\left(r, L_{b}, \widehat{\xi}_{2}\right)\right|_{\max }=g_{i_{1}}\left(r, L_{b}\right) \\
& +g_{i_{2}}\left(r, L_{b}\right)-f_{i_{1}, i_{2}}\left(r, \widehat{\xi}_{2}\right),
\end{aligned}
$$

where

$$
\begin{aligned}
f_{i_{1}, i_{2}} & \left(r, \widehat{\xi}_{2}\right) \\
& =g_{i_{1}}\left(r, \widehat{\xi}_{2}\right)+g_{i_{2}}\left(r, \widehat{\xi}_{2}\right) \\
& =\frac{\breve{M}}{\alpha}\left[(1-r) \frac{\phi_{i_{1}}\left(L_{b}\right)}{\omega_{i_{1}}} \phi_{i_{1}}^{\prime}\left(\widehat{\xi}_{2}\right)+r \frac{\phi_{i_{2}}\left(L_{b}\right)}{\omega_{i_{2}}} \phi_{i_{2}}^{\prime}\left(\widehat{\xi}_{2}\right)\right] .
\end{aligned}
$$

The ordinate $\widehat{\xi}_{2}$ of the absolute maximum of $\left|\widehat{w}\left(r, \xi_{1}, \xi_{2}\right)\right|$ will coincide with the absolute minimum of the $f_{i_{1}, i_{2}}\left(r, \xi_{2}\right)$. This position changes with the considered modes and the modes ratio $r$ so that the optimal location of the left edge of the PZT elements can be found by solving the following system of equations:

$$
\begin{aligned}
& \frac{\partial f_{i_{1}, i_{2}}\left(r, \xi_{2}\right)}{\partial \xi_{2}} \\
& =\frac{\breve{M}}{\alpha}\left[(1-r) \frac{\phi_{i_{1}}\left(L_{b}\right)}{\omega_{i_{1}}} \phi_{i_{1}}^{\prime \prime}\left(\xi_{2}\right)+r \frac{\phi_{i_{2}}\left(L_{b}\right)}{\omega_{i_{2}}} \phi_{i_{2}}^{\prime \prime}\left(\xi_{2}\right)\right]=0, \\
& \frac{\partial^{2} f_{i_{1}, i_{2}}\left(r, \xi_{2}\right)}{\partial \xi_{2}^{2}} \\
& =\frac{\breve{M}}{\alpha}\left[(1-r) \frac{\phi_{i_{1}}\left(L_{b}\right)}{\omega_{i_{1}}} \phi_{i_{1}}^{\prime \prime \prime}\left(\xi_{2}\right)+r \frac{\phi_{i_{2}}\left(L_{b}\right)}{\omega_{i_{2}}} \phi_{i_{2}}^{\prime \prime \prime}\left(\xi_{2}\right)\right]>0 .
\end{aligned}
$$

The solution will provide all the local minima and the absolute minimum will then be selected among these.

\section{Results and Discussions}

This section reports analytical results for different bimodal excitations and their comparison with FEM simulations. These simulations have been performed by using the frequency module of the structural mechanics module of COMSOL Multiphysics software [24]. Specifically, a steel beam of $30 \mathrm{~cm}$ of length has been taken into account; with bimodal control in mind and focusing on combinations of the first five modes, the wavelength of the highest mode has been divided into 50 subintervals of length $3 \mathrm{~mm}$, so that $\Delta a=\Delta h=3 \mathrm{~mm}$ and 5000 different combinations for $a$ and $h$ have been considered. For each of these, the amplitude of the response of the tip, to a periodic load, with the frequency corresponding to one of the first five eigenfrequencies has been calculated. Moreover the amplitude response to a linear combination of the previous loads has been obtained by superposition of the response to two of the eigenfrequencies. The optimal position has been chosen to be the one which corresponds to the maximum amplitude. In Figure $2 ; f_{1,2}\left(r, \bar{\xi}_{2}\right)$, the position of its absolute minimum, maximum and the position of the center of the piezoelectric plates, for different $r$ ratios, are reported. It is possible to observe that for $r=0$ and $r=1$ (that correspond, resp., to the excitation of the first and second modes) the optimal configuration of the PZT coincides with that obtained in [14]. For $r=0, f_{1,2}\left(0, \bar{\xi}_{2}\right)$ attains its absolute minimum at the boundary points $\widehat{\xi}_{2}=0$, so that the optimal configuration is with the piezoelectric plates distributed along the entire length of the beam. For increasing values of $r$ the position of the absolute minimum does not change until the derivative of $f_{i_{1}, i_{2}}\left(r, \bar{\xi}_{2}\right)$ becomes zero in $\bar{\xi}_{2}=0(25.1)$ :

$$
\widehat{r}=\frac{\phi_{1}^{\prime \prime}(0) \phi_{1}\left(L_{b}\right)}{\phi_{1}^{\prime \prime}(0) \phi_{1}\left(L_{b}\right)-\phi_{2}^{\prime \prime}(0) \phi_{2}\left(L_{b}\right)\left(\omega_{1} / \omega_{2}\right)} \cong 0.5 .
$$

For values of $r$ above $\widehat{r}$, the optimal configuration gradually changes to reach the optimal one for the second mode 


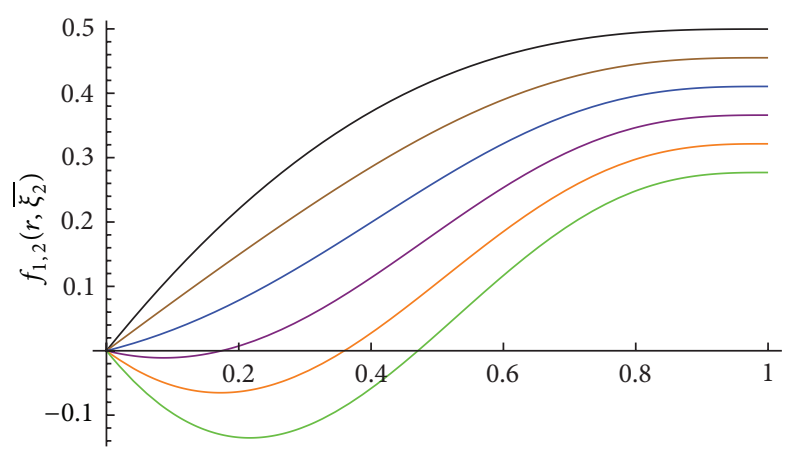

$\overline{\xi_{2}}$

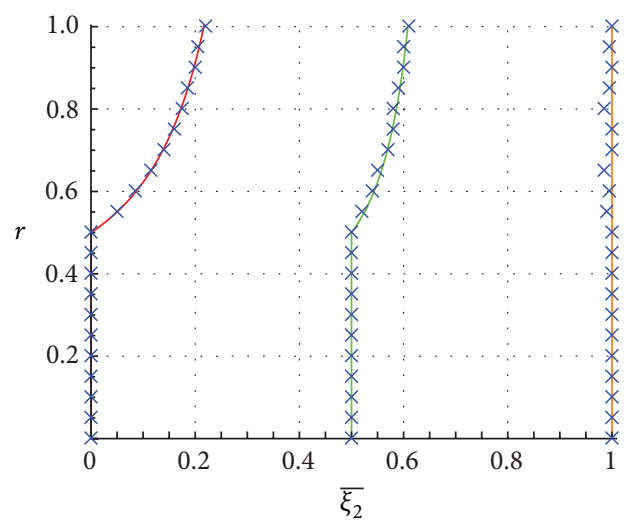

$$
\begin{aligned}
r & =0.0 \\
r & =0.2 \\
r & =0.4
\end{aligned} \quad \begin{aligned}
r & =0.6 \\
r & =0.8
\end{aligned}
$$

FIGURE 2: $f_{1,2}\left(r, \bar{\xi}_{2}\right)$; red line: PZT plate's left edge (analytical); green line: PZT position of the centre (analytical); orange line: PZT plate's right edge (analytical); $\times$ : FEM simulations.
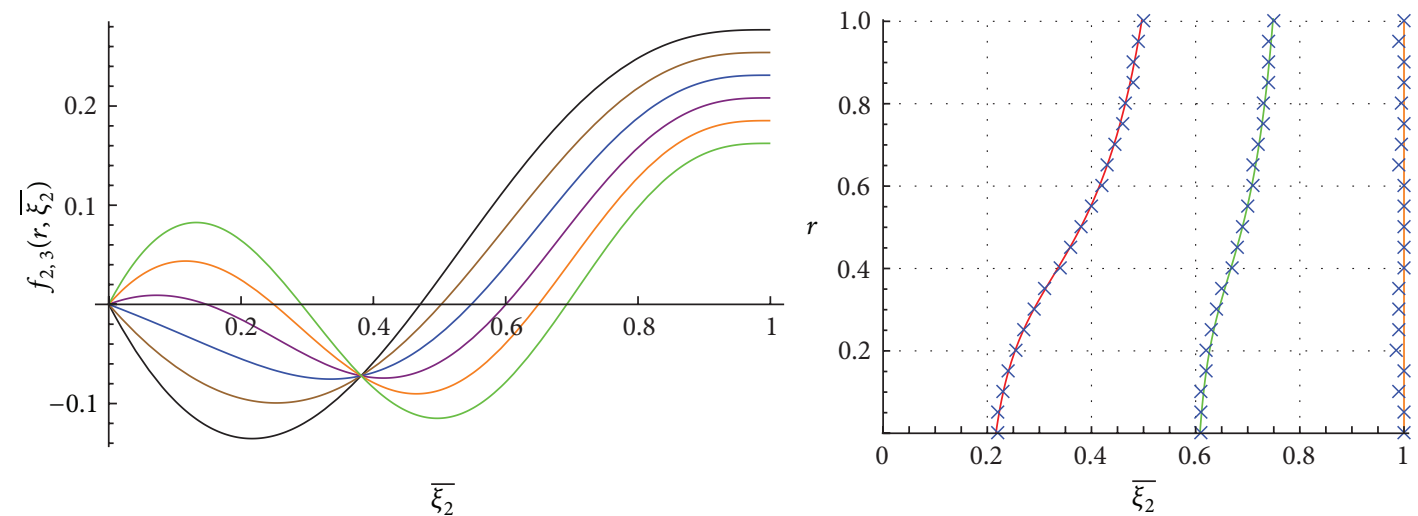

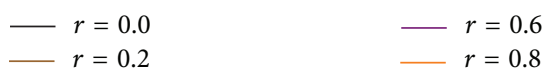

FIGURE 3: $f_{2,3}\left(r, \bar{\xi}_{2}\right)$; red line: PZT plate's left edge (analytical); green line: PZT position of the centre (analytical); orange line: PZT plate's right edge (analytical); $\times$ : FEM simulations.
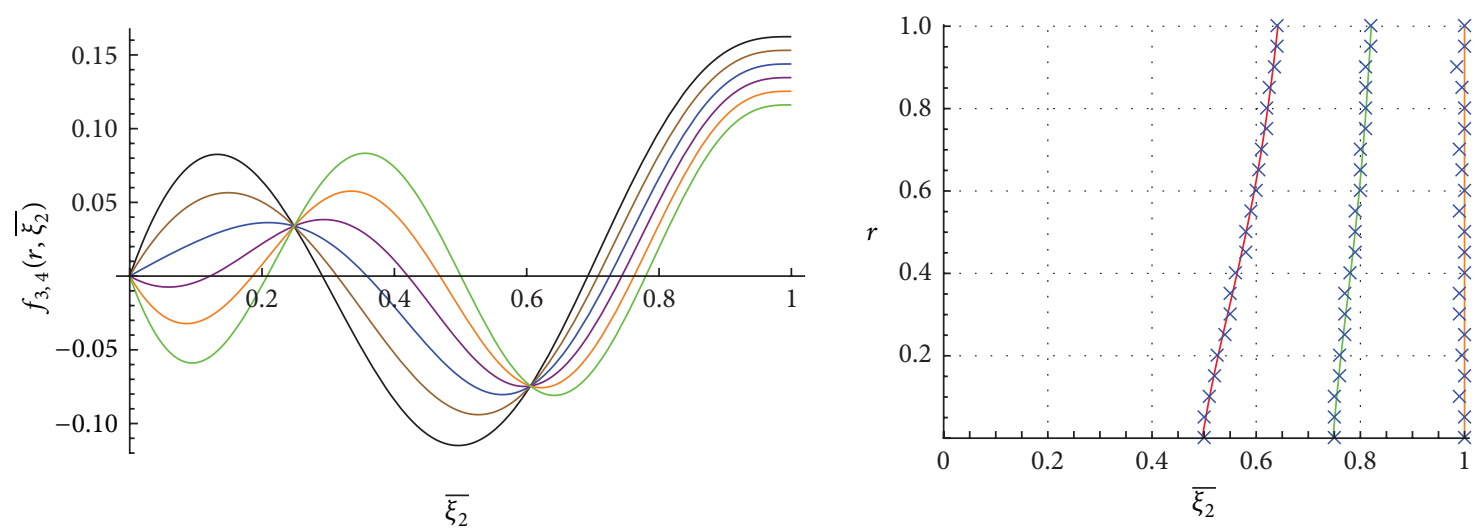

$$
\begin{aligned}
r & =0.0 \\
r & =0.2 \\
-r & =0.4
\end{aligned} \quad \begin{aligned}
r & =0.6 \\
r & =0.8 \\
r & =1.0
\end{aligned}
$$

Figure 4: $f_{3,4}\left(r, \bar{\xi}_{2}\right)$; red line: PZT plate's left edge (analytical); green line: PZT position of the centre (analytical); orange line: PZT plate's right edge (analytical); $\times$ : FEM simulations. 

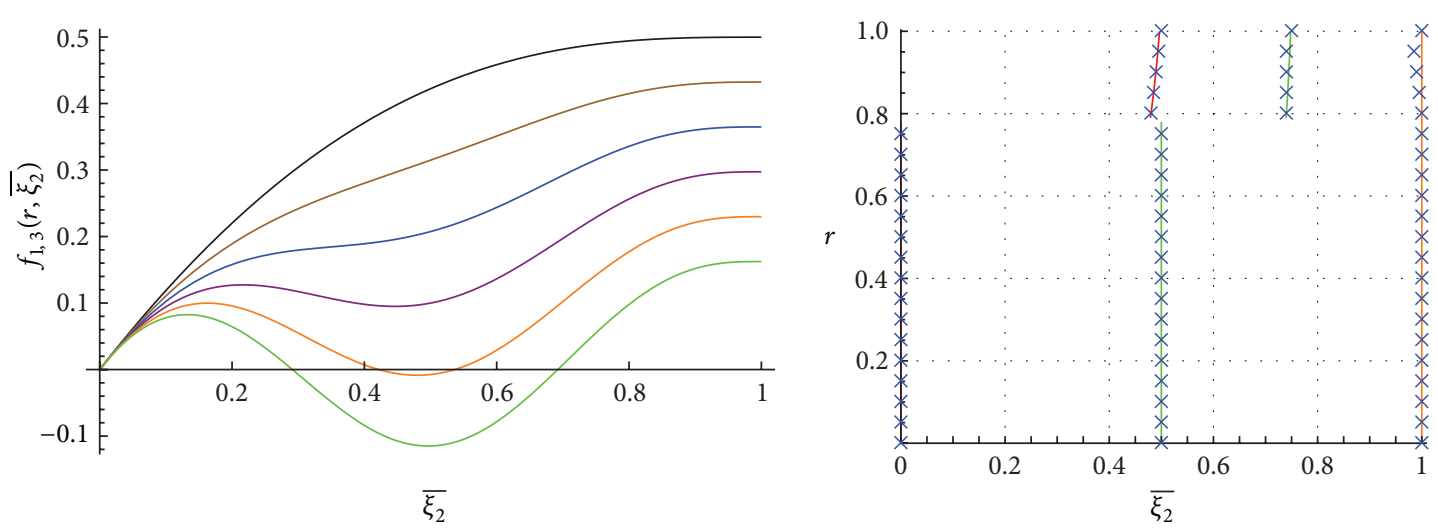

$$
\begin{array}{rlrl}
r & =0.0 \\
r & =0.2 \\
r & =0.4 & -r & =0.6 \\
-r & =0.8 \\
r & =1.0
\end{array}
$$

FIGURE 5: $f_{1,3}\left(r, \bar{\xi}_{2}\right)$; red line: PZT plate's left edge (analytical); green line: PZT position of the centre (analytical); orange line: PZT plate's right edge (analytical); $\times$ : FEM simulations.
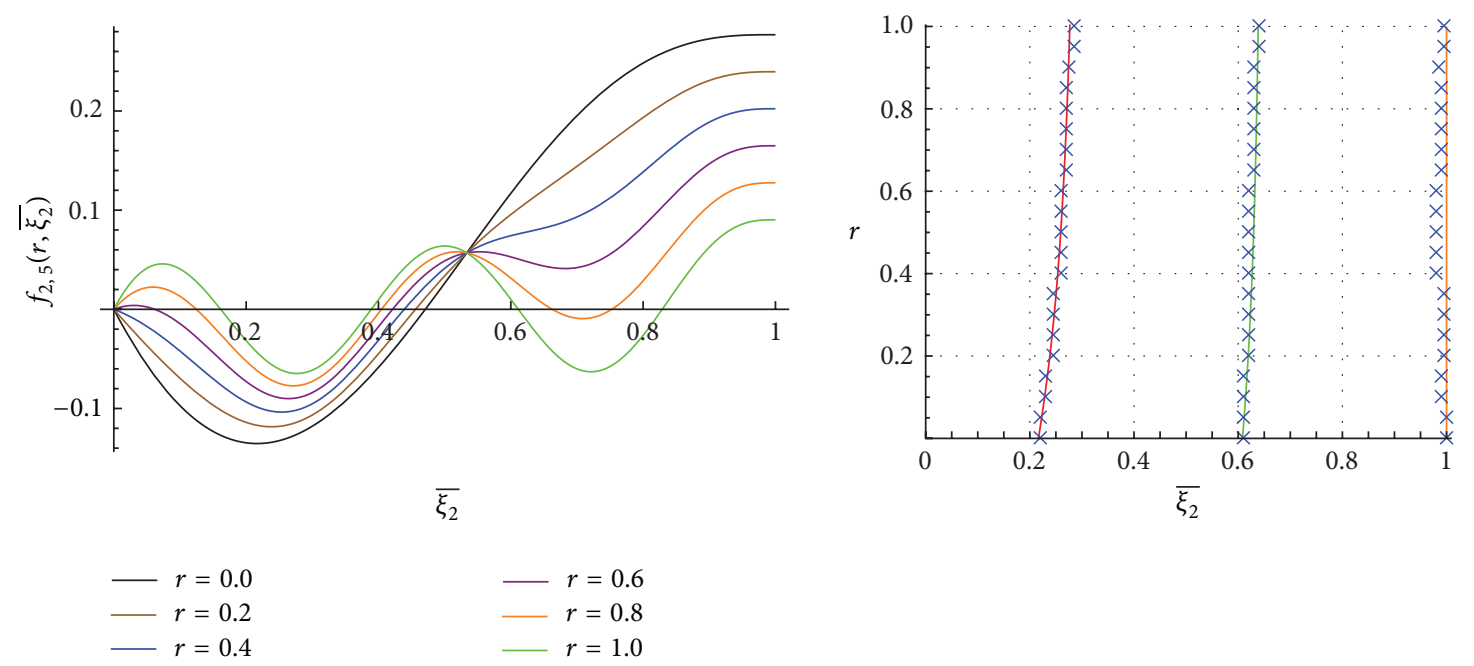

Figure 6: $f_{2,5}\left(r, \bar{\xi}_{2}\right)$; red line: PZT plate's left edge (analytical); green line: PZT position of the centre (analytical); orange line: PZT plate's right edge (analytical); $\times$ : FEM simulations.

at $r=1$. The FEM results show that there is a very good agreement between the numerical simulations and the analytical results. Different mode combinations are represented in Figures 3, 4, 5, 6, and 7: the optimal configuration varies from the optimal value for the individual $i_{1}$-excited mode $(r=0)$ to that for the mode $i_{2}(r=1)$. For all the considered coupled modes, the optimal position changes continuously except for the couple ((1)-(3)), where a different type of behaviour is observed (Figure 5 ), with a sharp transition at $r \cong 0.8$. This is due to the fact that, when $r$ is low, the absolute minimum of $f_{1,3}\left(r, \xi_{2}\right)$ coincides with that obtained for the optimal configuration of the first mode $(r=0)$. When the ratio reaches the value of $r \cong 0.46$, a relative minimum appears, but this develops into an absolute minimum and, therefore, coincides with the left end of the piezoelectric plates only when $r>0.8$; for such values the PZT elements should be active only over about half of the length of the beam. The comparisons with the FEM are in agreement with all the cases analyzed. The proposed analytical method has been also compared with the experimental investigations reported in [25-27]. To test different load spectra and configurations an experimental apparatus has been designed and built; moreover, a MATLAB code has been developed to control the actuation and the modal contributions. Four piezoelectric plates have been used in three different configurations. Experiments have been done with one mode and multimodal vibrations. A good agreement was shown in all the considered cases.

\section{Conclusions}

In this work a new theoretical model for the optimal placement of piezoelectric plates to control the multimode 

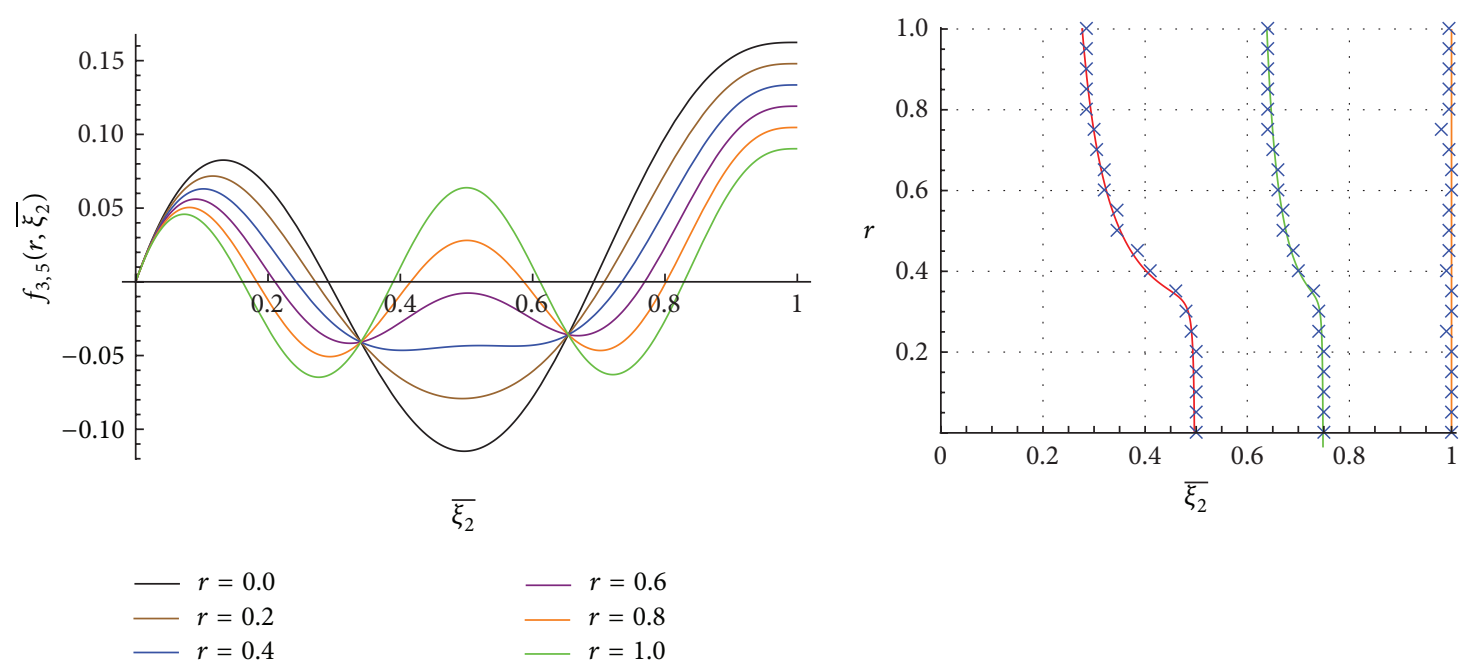

FIgure 7: $f_{3,5}\left(r, \bar{\xi}_{2}\right)$; red line: PZT plate's left edge (analytical); green line: PZT position of the centre (analytical); orange line: PZT plate's right edge (analytical); $\times$ : FEM simulations.

vibrations of a cantilever beam is proposed. After a detailed description of the theoretical model, bi-modal excitation is considered. The designs of the optimal configurations, for different coupled modes and different relative contributions, are reported. The comparison between numerical and theoretical results is shown to confirm the validity of the optimization technique. This model can be very useful in many real situations when the spectrum of the load excites more eigenmodes and has, therefore, implications for the development of strategies to control multimode vibrations. This methodology has been extended to rotating systems [28, 29]. Its application to real systems subjected to the centrifugal loads, for example, gas turbine blades [30], and impulse loads [31] will constitute the object of further contributions.

\section{Nomenclature}

a: Position of the center of the piezoelectric plates

B: Control vector

c: Beam width

C: Damping matrix

$d_{31}$ : Piezoelectric coefficient

$E_{a}$ : Young's modulus of the piezoelectric material

$E_{b}$ : Young's modulus of the beam material

$h$ : Piezoelectric plates length

$I_{b}$ : Inertia moment of the beam

K: Stiffness matrix

$L_{b}$ : Beam length

$M$ : Piezoelectric bending moment

M: Mass matrix

$r$ : Percentage coupling coefficient

$S: \quad$ Cross-section area of the beam

$T_{a}$ : Piezoelectric thickness

$T_{b}$ : Beam thickness

$V$ : Voltage applied to the piezoelectric plates

$w$ : Vertical displacement

$\widetilde{w}$ : Virtual vertical displacement $\alpha$ : Damping coefficient

$\phi_{i}(x)$ : $i$ th flexural mode of the cantilever beam

$\rho: \quad$ Mass density

$X_{i}(t)$ : Amplitude of the $i$ th mode

$\omega_{i}$ : Natural frequency.

\section{References}

[1] E. Poursaeidi and M. Salavatian, "Fatigue crack growth simulation in a generator fan blade," Engineering Failure Analysis, vol. 16, no. 3, pp. 888-898, 2009.

[2] L. Witek, "Experimental crack propagation and failure analysis of the first stage compressor blade subjected to vibration," Engineering Failure Analysis, vol. 16, no. 7, pp. 2163-2170, 2009.

[3] J. Kubiak Sz, G. Urquiza B, J. García C, and F. Sierra E, "Failure analysis of steam turbine last stage blade tenon and shroud," Engineering Failure Analysis, vol. 14, no. 8, pp. 1476-1487, 2007.

[4] E. F. Crawley and J. de Luis, "Use of piezoelectric actuators as elements of intelligent structures," AIAA Journal, vol. 25, no. 10, pp. 1373-1385, 1987.

[5] F. Botta and G. Cerri, "Wave propagation in Reissner-Mindlin piezoelectric coupled cylinder with non-constant electric field through the thickness," International Journal of Solids and Structures, vol. 44, no. 18-19, pp. 6201-6219, 2007.

[6] M. I. Frecker, "Recent advances in optimization of smart structures and actuators," Journal of Intelligent Material Systems and Structures, vol. 14, no. 4-5, pp. 207-216, 2003.

[7] V. Gupta, M. Sharma, and N. Thakur, "Optimization criteria for optimal placement of piezoelectric sensors and actuators on a smart structure: a technical review," Journal of Intelligent Material Systems and Structures, vol. 21, no. 12, pp. 1227-1243, 2010.

[8] K. D. Dhuri and P. Seshu, "Piezo actuator placement and sizing for good control effectiveness and minimal change in original system dynamics," Smart Materials and Structures, vol. 15, no. 6, pp. 1661-1672, 2006.

[9] K. R. Kumar and S. Narayanan, "Active vibration control of beams with optimal placement of piezoelectric sensor/actuator 
pairs," Smart Materials and Structures, vol. 17, no. 5, Article ID 055008, 2008.

[10] M. Sunar and S. S. Rao, "Distributed modeling and actuator location for piezoelectric control systems," AIAA Journal, vol. 34, no. 10, pp. 2209-2211, 1996.

[11] M. A. Demetriou, "Numerical algorithm for the optimal placement of actuators and sensors for flexible structures," in Proceedings of the American Control Conference, pp. 2290-2294, Chicago, Ill, USA, June 2000.

[12] I. Bruant, G. Coffignal, F. Lene, and M. Verge, "A methodology for determination of piezoelectric actuator and sensor location on beam structures," Journal of Sound and Vibration, vol. 243, no. 5, pp. 861-882, 2001.

[13] Y. Yang, Z. Jin, and C. K. Soh, "Integrated optimal design of vibration control system for smart beams using genetic algorithms," Journal of Sound and Vibration, vol. 282, no. 3-5, pp. 1293-1307, 2005.

[14] R. Barboni, A. Mannini, E. Fantini, and P. Gaudenzi, "Optimal placement of PZT actuators for the control of beam dynamics," Smart Materials and Structures, vol. 9, no. 1, pp. 110-120, 2000.

[15] O. J. Aldraihem, T. Singh, and R. C. Wetherhold, "Optimal size and location of piezoelectric actuator/sensors: practical considerations," Journal of Guidance, Control, and Dynamics, vol. 23, no. 3, pp. 509-515, 2000.

[16] A. Baz and S. Poh, "Performance of an active control system with piezoelectric actuators," Journal of Sound and Vibration, vol. 126, no. 2, pp. 327-343, 1988.

[17] S. M. Yang and Y. J. Lee, "Optimization of non-collocated sensor/actuator location and feedback gain in control systems," Smart Materials and Structures, vol. 2, no. 2, pp. 96-102, 1993.

[18] S. M. Yang and Y. J. Lee, "Vibration suppression with optimal sensor/actuator location and feedback gain," Smart Materials and Structures, vol. 2, no. 4, pp. 232-239, 1993.

[19] Q. Wang and C. M. Wang, "Optimal placement and size of piezoelectric patches on beams from the controllability perspective," Smart Materials and Structures, vol. 9, no. 4, pp. 558-567, 2000.

[20] A. Hohl, M. Neubauer, S. M. Schwarzendahl, L. Panning, and J. Wallaschek, "Active and semiactive vibration damping of turbine blades with piezoceramics," in Active and Passive Smart Structures and Integrated Systems, vol. 7288 of Proceedings of SPIE, San Diego, Calif, USA, March 2009.

[21] I. Goltz, H. Bhmer, R. Nollau, J. Belz, B. Grueber, and J. R. Seume, "Piezo-electric actuation of rotor blades in an axial compressor with piezoceramics," in Proceedings of 8th European Conference on Turbomachinery (ETC '09), Graz, Austria, March 2009.

[22] A. J. Provenza and C. R. Morrison, "Control of fan blade vibrations using piezo-electric and bi-directional telemetry," in Proceedings of the ASME Turbo Expo, Vancouver, Canada, June 2011.

[23] S. M. Lin, I. C. Mao, and J. H. Lin, "Vibration of a rotating smart beam," AIAA Journal, vol. 45, no. 2, pp. 382-389, 2007.

[24] Comsol Multiphysics User's Guide Version 4.3.

[25] F. Botta, N. Marx, S. Gentili et al., "Optimal placement of piezoelectric plates for active vibration control of gas turbine blades: experimental results," in Sensors and Smart Structures Technologies for Civil, Mechanical, and Aerospace Systems, vol. 8345 of Proceedings of SPIE, San Diego, Calif, USA, March 2012.

[26] F. Botta, N. Marx, D. Dini, R. de Lieto Vollaro, and G. Battista, "Experimental results for optimal placement of piezoelectric plates for active vibration control of a cantilever beam," International Journal of Engineering and Technology, vol. 5, no. 5, 2013.

[27] N. Marx, Counter vibration on gas turbine compressor blades via piezoelectric plates [M.S. thesis], Imperial College, London, UK, 2012.

[28] F. Botta, N. Marx, C. W. Schwingshackl, G. Cerri, and D. Dini, "A wireless vibration control technique for gas turbine blades using piezoelectric plates and contactless energy transfer," in Proceedings of the ASME Turbo Expo, San Antonio, Tex, USA, June 2013.

[29] F. Botta, D. Dini, and R. de Lieto Vollaro, "A new function for the optimal placement of piezoelectric plates to control multimode vibrations of a rotating beam," International Journal of Engineering and Technology, vol. 5, no. 5, 2013.

[30] G. Cerri, M. Gazzino, F. Botta, and C. Salvini, "Production planning with hot section life prediction for optimum gas turbine management," International Journal of Gas Turbine, Propulsion and Power Systems, vol. 2, no. 1, pp. 9-16, 2008.

[31] F. Botta and G. Cerri, "Shock response spectrum in plates under impulse loads," Journal of Sound and Vibration, vol. 308, no. 3-5, pp. 563-578, 2007. 

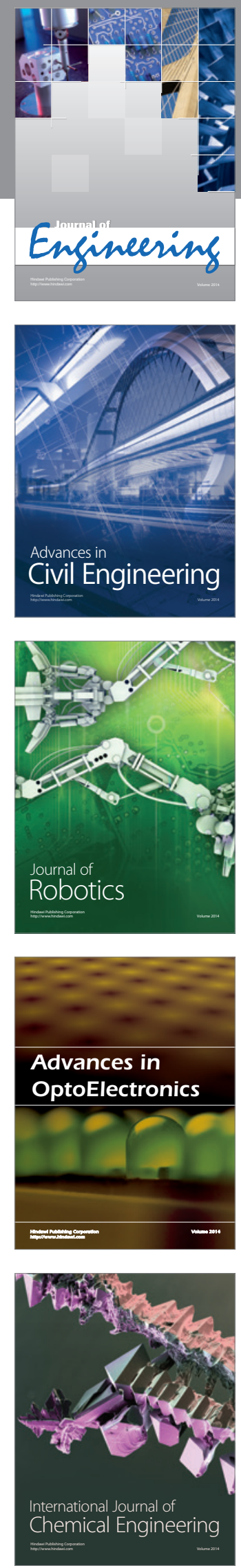

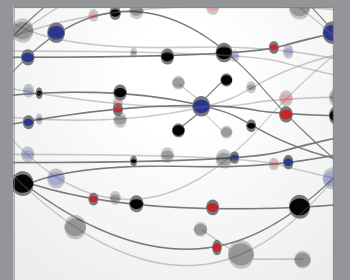

The Scientific World Journal
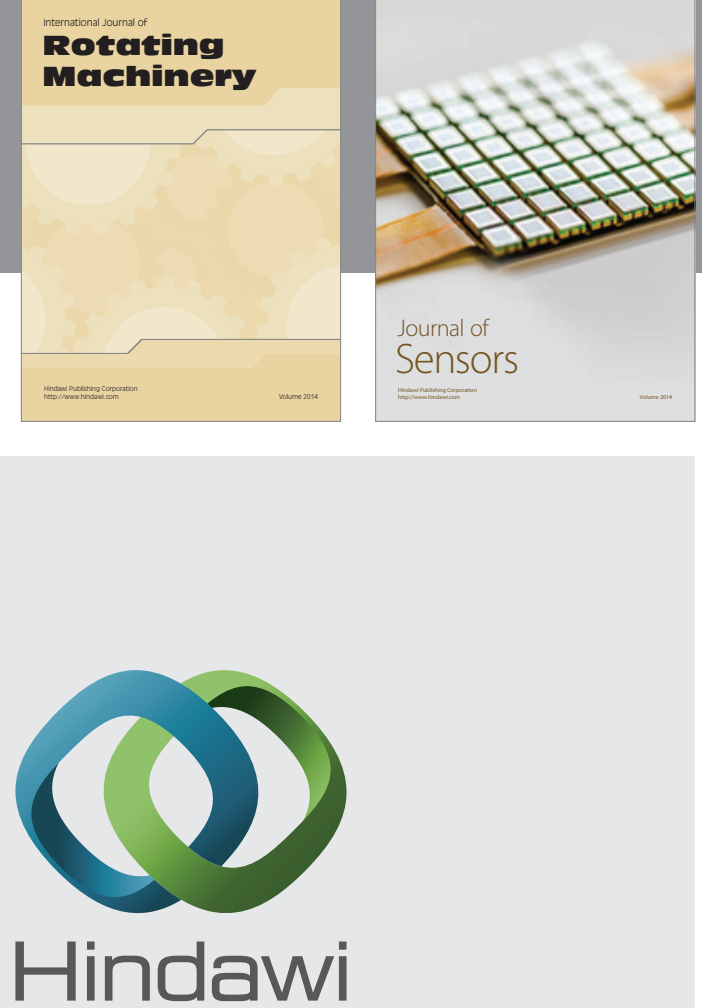

Submit your manuscripts at http://www.hindawi.com
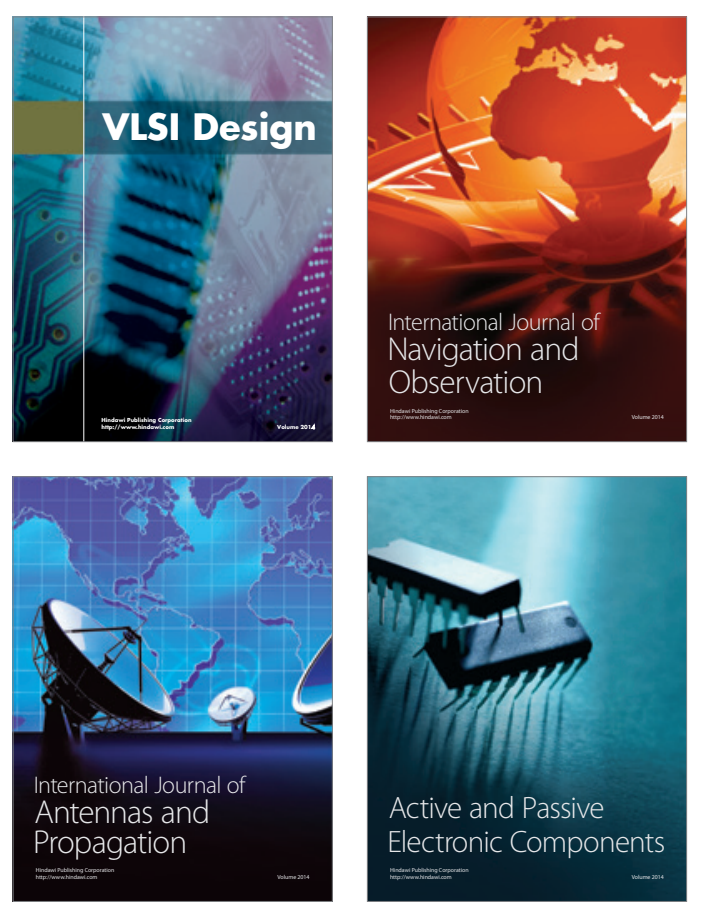
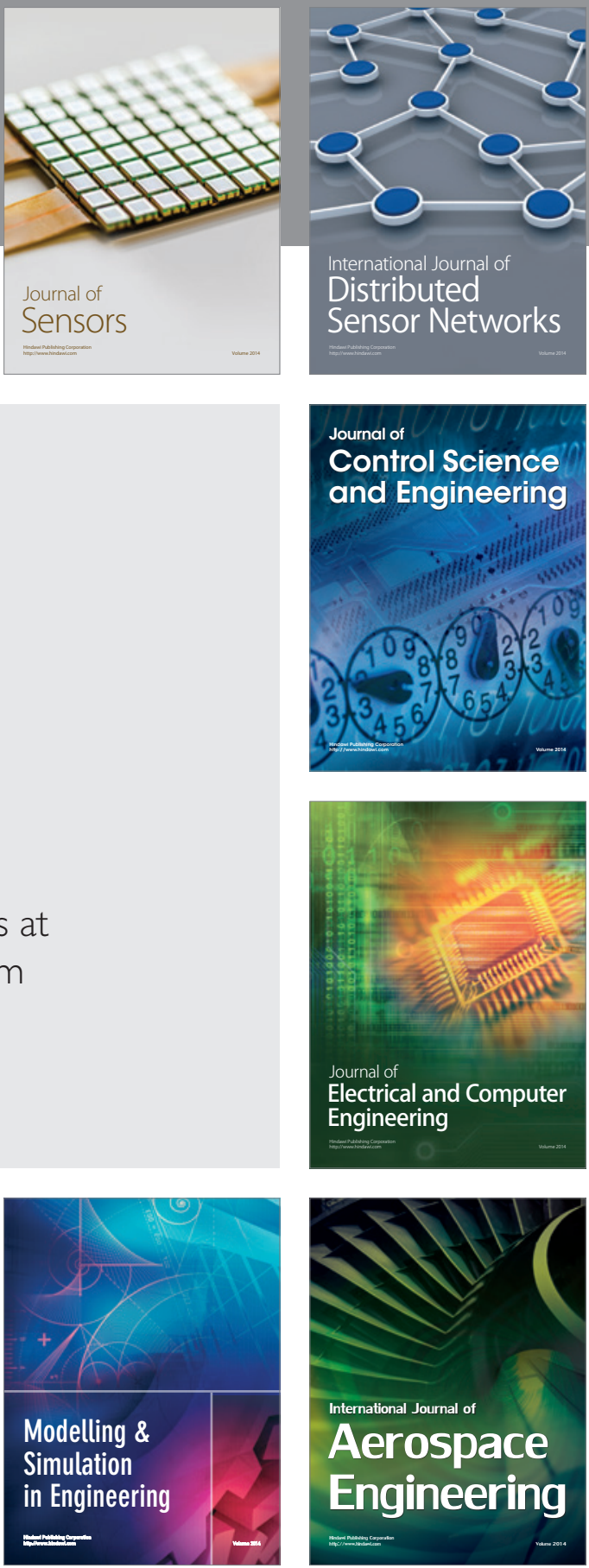

Journal of

Control Science

and Engineering
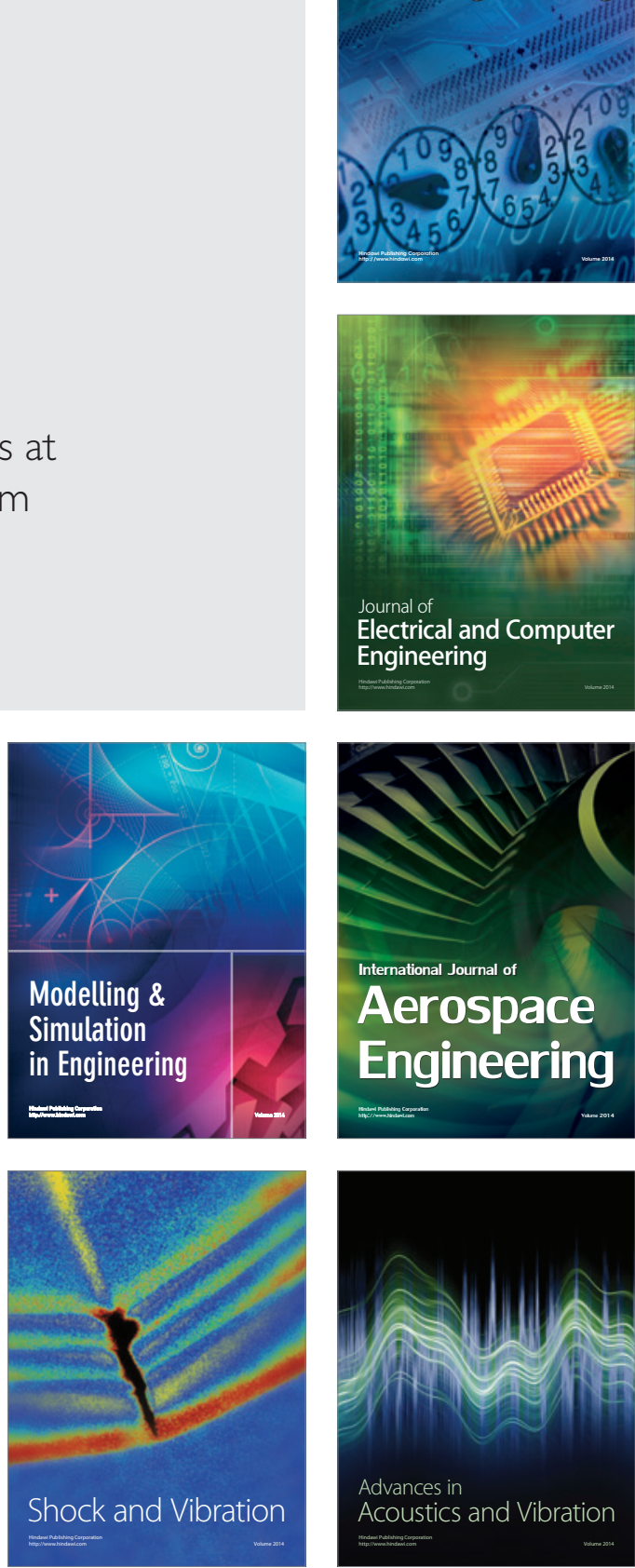